\title{
ON HOMOLOGICAL PROPERTIES FOR SOME MODULES OF UNIFORMLY CONTINUOUS FUNCTIONS OVER CONVOLUTION ALGEBRAS
}

\author{
RASOUL NASR-ISFAHANI ${ }^{\bowtie}$ and SIMA SOLTANI RENANI
}

(Received 22 January 2010)

\begin{abstract}
For a locally compact group $G$, let $\operatorname{LUC}(G)$ denote the space of all left uniformly continuous functions on $G$. Here, we investigate projectivity, injectivity and flatness of $\operatorname{LUC}(G)$ and its dual space $\operatorname{LUC}(G)^{*}$ as Banach left modules over the group algebra as well as the measure algebra of $G$.
\end{abstract}

2010 Mathematics subject classification: primary 43A07; secondary 43A15, 43A20, 46H05, 46H25.

Keywords and phrases: amenability, flatness, injectivity, projectivity, Banach module, locally compact group.

\section{Introduction and preliminaries}

Let $G$ denote a locally compact group with identity element $e$, modular function $\Delta$, and a fixed left Haar measure $\lambda$. As usual, let $M(G)$ denote the measure algebra of $G$ as defined in [4], endowed with the total variation norm $\|\cdot\|$ and the convolution product of measures, and let $\delta_{x} \in M(G)$ denote the Dirac measure at $x \in G$. Let also $L^{1}(G)$ denote the group algebra of $G$ as defined in [4], equipped with the norm $\|\cdot\|_{1}$ and the convolution product $*$ of functions on $G$. Then $L^{1}(G)$ is a Banach $M(G)$ bimodule with the module actions defined by

$$
(\phi \cdot \mu)(x)=\int_{G} \phi\left(x y^{-1}\right) \Delta\left(y^{-1}\right) d \mu(y)
$$

and

$$
(\mu \cdot \phi)(x)=\int_{G} \phi\left(y^{-1} x\right) d \mu(y)
$$

for all $\phi \in L^{1}(G), \mu \in M(G)$ and locally almost all $x \in G$. Now, let $L^{\infty}(G)$ denote the Lebesgue space as defined in [4], equipped with the essential supremum norm $\|\cdot\|_{\infty}$. Then $L^{\infty}(G)$ is the dual bimodule of the Banach $M(G)$-bimodule $L^{1}(G)$ under

(C) 2011 Australian Mathematical Publishing Association Inc. 0004-9727/2011 \$16.00 
the pairing

$$
\langle f, \phi\rangle=\int_{G} f(x) \phi(x) d \lambda(x)
$$

for all $\phi \in L^{1}(G)$ and $f \in L^{\infty}(G)$. We denote by $\operatorname{LUC}(G)$ the space of all bounded left uniformly continuous functions on $G$ and by $C_{0}(G)$ the space of all continuous functions on $G$ vanishing at infinity. Then $\operatorname{LUC}(G)$ and $C_{0}(G)$ are closed submodules of the Banach $M(G)$-bimodule $L^{\infty}(G)$. Hence, the dual spaces $\operatorname{LUC}(G)^{*}$ of $\operatorname{LUC}(G)$ and $M(G)$ of $C_{0}(G)$ are Banach $M(G)$-bimodules with the dual actions; the $M(G)$ module actions of $M(G)$ are just the convolution product in $M(G)$.

In particular, $L^{\infty}(G)$ is a Banach $L^{1}(G)$-bimodule for which the left and right module actions of $L^{1}(G)$ on $L^{\infty}(G)$ are given by the formulae

$$
\phi \cdot f=f * \widetilde{\phi} \quad \text { and } \quad f \cdot \phi=\frac{1}{\Delta} \widetilde{\phi} * f
$$

for all $f \in L^{\infty}(G)$ and $\phi \in L^{1}(G)$, where

$$
\widetilde{\phi}(x)=\phi\left(x^{-1}\right)
$$

for all $x \in G$. Moreover, $\operatorname{LUC}(G)$ and $C_{0}(G)$ are closed submodules of the Banach $L^{1}(G)$-bimodule $L^{\infty}(G)$. Thus, $\operatorname{LUC}(G)^{*}$ is a Banach $L^{1}(G)$-bimodule with the dual actions

$$
\langle\phi \cdot H, h\rangle=\left\langle H, \frac{1}{\Delta} \widetilde{\phi} * h\right\rangle
$$

and

$$
\langle H \cdot \phi, h\rangle=\langle H, h * \widetilde{\phi}\rangle
$$

for all $\phi \in L^{1}(G), h \in \operatorname{LUC}(G)$ and $H \in \operatorname{LUC}(G)^{*}$. Similarly, $M(G)$ is a Banach $L^{1}(G)$-bimodule. Finally, we identify $L^{1}(G)$ with a closed ideal of $M(G)$ and consider $L^{1}(G)$ as a closed submodule of $M(G)$ whose module actions are the same as the convolution product $*$.

Dales and Polyakov [2] have characterized projectivity, injectivity and flatness of certain Banach left $L^{1}(G)$-modules; see also the recent works [1, 7]. In this work, we intend to characterize these homological properties for $\operatorname{LUC}(G)$ and $\operatorname{LUC}(G)^{*}$ as Banach left $L^{1}(G)$-modules and $M(G)$-modules in terms of some topological and algebraic properties of $G$.

\section{The results}

For two Banach spaces $E$ and $F$, let $B(E, F)$ denote the Banach space of all bounded operators from $E$ into $F$. An operator $T \in B(E, F)$ is called admissible if

$$
T \circ S \circ T=T
$$

for some $S \in B(F, E)$. In the case where $A$ is a Banach algebra and $E$ and $F$ are Banach left $A$-modules, ${ }_{A} B(E, F)$ denotes the closed linear subspace of $B(E, F)$ of 
all left $A$-module morphisms. An operator $T \in{ }_{A} B(E, F)$ is a retraction if there exists $S \in{ }_{A} B(F, E)$ with

$$
T \circ S=I_{F},
$$

the identity operator on $F$; in this case, $F$ is called a retract of $E$. A Banach left $A$-module $P$ is called projective if for each Banach left $A$-modules $E$ and $F$, each admissible epimorphism $T \in{ }_{A} B(E, F)$ and each $S \in{ }_{A} B(P, F)$, there exists $R \in{ }_{A} B(P, E)$ such that

$$
T \circ R=S .
$$

We can now state our first result which characterizes projectivity of $\operatorname{LUC}(G)^{*}$ as a Banach left $L^{1}(G)$-module.

THEOREM 2.1. Let $G$ be a locally compact group. If $L U C(G)^{*}$ is a projective Banach left $L^{1}(G)$-module, then $G$ is discrete and contains no infinite amenable subgroup.

Proof. Suppose that $\operatorname{LUC}(G)^{*}$ is a projective Banach left $L^{1}(G)$-module. On the one hand, $M(G)$ is a projective Banach left $L^{1}(G)$-module if and only if $G$ is discrete; see [2, Theorem 2.6]. On the other hand, each retraction of a projective Banach left $L^{1}(G)$-module is projective; see [3]. To show that $G$ is discrete we only need to prove that $M(G)$ is a retraction of the Banach left $L^{1}(G)$-module $\operatorname{LUC}(G)^{*}$.

To that end, define $\mathcal{Q}: M(G) \longrightarrow \operatorname{LUC}(G)^{*}$ to be the map that sends a measure $\mu$ in $M(G)$ to the integration functional

$$
h \mapsto \int_{G} h d \mu
$$

for all $h \in \operatorname{LUC}(G)$. This is well defined because $h$ is continuous and corresponds to choosing the extension $u$ of $\delta_{e}$ to be the functional on $\operatorname{LUC}(G)$ that evaluates a function at $e \in G$; in fact, $\mathcal{Q}(\mu)=\mu \cdot u$ for all $\mu \in M(G)$. Clearly, $\mathcal{Q}$ is a left $L^{1}(G)$-module morphism. Now, let

$$
\mathcal{P}: \operatorname{LUC}(G)^{*} \longrightarrow M(G)
$$

be the restriction map, and note that $\mathcal{P}$ is a left $L^{1}(G)$-module morphism. One can easily check that for every $\mu \in M(G)$ and $h \in C_{0}(G)$, the function $h \cdot \mu \in C_{0}(G)$ is given by

$$
(h \cdot \mu)(x)=\int_{G} h(y x) d \mu(y)
$$

for all $x \in G$, and we therefore have

$$
\langle\mu \cdot u, h\rangle=\langle u, h \cdot \mu\rangle=(h \cdot \mu)(e)=\langle\mu, h\rangle .
$$

That is, $\mathcal{Q}$ is a right inverse for $\mathcal{P}$, and thus $M(G)$ is a retraction of $\operatorname{LUC}(G)^{*}$.

In particular, since we now know that $G$ is discrete,

$$
\operatorname{LUC}(G)^{*}=L^{\infty}(G)^{*} .
$$

So, the second part follows from the fact that $G$ contains no infinite amenable subgroup if $L^{\infty}(G)^{*}$ is a projective Banach left $L^{1}(G)$-module; see [2, Theorem 2.7]. 
Let $A$ be a Banach algebra and $E$ be a Banach left $A$-module. Then the space $A \widehat{\otimes} E$ is a Banach left $A$-module with the action $a \cdot(b \otimes \xi)=a b \otimes \xi$ for all $a, b \in A$ and $\xi \in E$. Define the left $A$-module morphism $\pi: A \widehat{\otimes} E \longrightarrow E$ by the formula

$$
\pi(a \otimes \xi)=a \cdot \xi
$$

for $\xi \in E$ and $a \in A$. It is shown in [3, Proposition IV.1.1], that if $E$ is essential as a Banach left $A$-module (that is, the linear span of $A \cdot E$ is dense in $E$ ), then $E$ is projective if and only if the canonical morphism $\pi \in{ }_{A} B(A \widehat{\otimes} E, E)$ is a retraction.

THEOREM 2.2. Let $G$ be a compact group. Then $L U C(G)^{*}$ is a projective Banach left $M(G)$-module.

Proof. It is clear that if $G$ is compact, then $\operatorname{LUC}(G)=C_{0}(G)$ and so $\operatorname{LUC}(G)^{*}=$ $M(G)$. So, the result follows from the fact that $M(G)$ is always a projective Banach left $M(G)$-module; indeed, if $\delta_{e}$ is the Dirac measure at $e \in G$, then the left $M(G)$ module morphism

$$
\rho: \mu \mapsto \mu \otimes \delta_{e}, \quad M(G) \rightarrow M(G) \widehat{\otimes} M(G),
$$

is a right inverse for the canonical morphism $\pi: M(G) \widehat{\otimes} M(G) \rightarrow M(G)$.

We conjecture that the converse of Theorem 2.2 is also true. In proving the next result, we need the following consequence from [3, Corollary IV.4.5]. But first we recall that a Banach space $E$ has approximation property if the identity operator on $E$ can be approximated in the compact-open topology by finite dimensional operators.

Proposition 2.3. Let $A$ be a Banach algebra and $E$ be a Banach left A-module. Suppose that one of $A$ and $E$ has approximation property. Then for each $\xi \in E \backslash\{0\}$, there is $T \in{ }_{A} B\left(E, A^{b}\right)$ such that $T(\xi) \neq 0$, where $A^{b}$ is the unitization of $A$. In the case where $E$ is essential, we may suppose that $T \in{ }_{A} B(E, A)$.

Using this result, we describe projectivity of $\operatorname{LUC}(G)$ as a Banach left $L^{1}(G)$ - or $M(G)$-module.

THEOREM 2.4. Let $G$ be a locally compact group. Then the following statements are equivalent.

(a) $L U C(G)$ is a projective Banach left $L^{1}(G)$-module.

(b) $L U C(G)$ is a projective Banach left $M(G)$-module.

(c) $G$ is compact.

Proof. (a) $\Rightarrow\left(\right.$ b). Suppose that $\operatorname{LUC}(G)$ is a projective Banach left $L^{1}(G)$ module. Then $\operatorname{LUC}(G)$ is a projective Banach left $M(G)$-module if we show that for each pair of Banach left $M(G)$-modules $E$ and $F$, each admissible epimorphism $T \in \in_{M(G)} B(E, F)$ and each $S \in{ }_{M(G)} B(\operatorname{LUC}(G), F)$ there exists $R \in$ $M(G) B(\operatorname{LUC}(G), E)$ such that

$$
T \circ R=S
$$


Since $\operatorname{LUC}(G)$ is a projective Banach left $L^{1}(G)$-module, there exists a left $L^{1}(G)$ morphism $R: \operatorname{LUC}(G) \longrightarrow E$ with

$$
T \circ R=S .
$$

To that end, we only need to show that $R$ is a left $M(G)$-morphism. Choose a bounded approximate identity $\left(e_{\gamma}\right)_{\gamma \in \Gamma}$ in $L^{1}(G)$, and note that $\left(e_{\gamma}\right)_{\gamma \in \Gamma}$ is a left approximate identity for the Banach left $L^{1}(G)$-module $\operatorname{LUC}(G)$. Then for each $\mu \in M(G)$ and $h \in \operatorname{LUC}(G)$,

$$
\begin{aligned}
R(\mu \cdot h) & =\lim _{\gamma} R\left(\mu \cdot e_{\gamma} \cdot h\right) \\
& =\lim _{\gamma} \mu \cdot e_{\gamma} \cdot R(h) \\
& =\lim _{\gamma} \mu \cdot R\left(e_{\gamma} \cdot h\right) \\
& =\mu \cdot R\left(\lim _{\gamma} e_{\gamma} \cdot h\right) \\
& =\mu \cdot R(h) .
\end{aligned}
$$

(b) $\Rightarrow$ (c). Suppose that $\operatorname{LUC}(G)$ is projective as a Banach left $M(G)$-module, and let $\varphi \in \operatorname{LUC}(G)$ be a function with compact support such that $0 \leq \varphi \leq 1$ and $\varphi(e)=1$. Since $\operatorname{LUC}(G)$ is an essential Banach left $M(G)$-module and $M(G)$ has the approximation property, it follows from Proposition 2.3 that there exists a left $M(G)$-module morphism $T: \operatorname{LUC}(G) \longrightarrow M(G)$ such that

$$
T(\varphi) \neq 0 .
$$

We may suppose that $T(\operatorname{LUC}(G)) \subseteq L^{1}(G)$; otherwise, we replace $T$ by the map

$$
h \longmapsto T(h) \cdot \phi
$$

from $\operatorname{LUC}(G)$ into $L^{1}(G)$ for some function $\phi \in L^{1}(G)$ with $T(\varphi) \cdot \phi \neq 0$. Choose a natural number $n$ with

$$
2(\|T\|+1)<n\|T(\varphi)\|_{1} .
$$

Then there is a continuous function $\psi$ with compact support such that

$$
n\|T(\varphi)-\psi\|_{1}<1
$$

In particular, $\|T(\varphi)\|_{1}<2\|\psi\|_{1}$.

Now, suppose on the contrary that $G$ is not compact. Then there exists $x_{1}, \ldots$, $x_{n} \in G$ such that the sets $x_{i} C$ are pairwise disjoint for $i=1, \ldots, n$, where

$$
C:=\operatorname{supp}(\varphi) \cup \operatorname{supp}(\psi) .
$$

So, if we put $\mu=\delta_{x_{1}}+\cdots+\delta_{x_{n}}$, then

$$
\|\mu * \varphi\|_{\infty}=1 \quad \text { and } \quad\|\mu * \psi\|_{1}=n\|\psi\|_{1} .
$$


Thus

$$
\begin{aligned}
n\|\psi\|_{1} & =\|\mu * \psi\|_{1} \\
& \leq\|T(\mu * \varphi)\|_{1}+\|\mu *(T(\varphi)-\psi)\|_{1} \\
& \leq\|T\|\|\mu * \varphi\|_{\infty}+n\|T(\varphi)-\psi\|_{1} \\
& =\|T\|+1 \\
& <\frac{n}{2}\|T(\varphi)\|_{1} .
\end{aligned}
$$

So, $\|T(\varphi)\|_{1} \geq 2\|\psi\|_{1}$. This contradiction completes the proof.

(b) $\Rightarrow$ (c). This is proved in [2, Theorem 3.1].

Let $A$ be a Banach algebra. A Banach left $A$-module $I$ is called injective if for each Banach left $A$-modules $E$ and $F$, each admissible monomorphism $T \in{ }_{A} B(E, F)$ and each $S \in{ }_{A} B(E, I)$, there exists $R \in{ }_{A} B(F, I)$ such that

$$
R \circ T=S .
$$

Similar definitions apply for Banach right $A$-modules.

For each Banach left $A$-module $E$, the space $B(A, E)$ is a Banach left $A$-module with

$$
(a \cdot T)(b)=T(b a)
$$

for all $a, b \in A$ and $T \in B(A, E)$. Define the left $A$-module morphism $\Pi: E \longrightarrow$ $B(A, E)$ by the formula

$$
\Pi(\xi)(a)=a \cdot \xi
$$

for $\xi \in E$ and $a \in A$. It is shown in [3, Proposition III.1.31], that if $A$ is a Banach algebra, and $E$ is faithful as a Banach left $A$-module (that is, $A \cdot \xi \neq\{0\}$ for all $\xi \in E \backslash\{0\})$, then $E$ is injective if and only if there exists a left $A$-module morphism $\rho: B(A, E) \longrightarrow E$ with

$$
\rho \circ \Pi=I_{E} .
$$

THEOREM 2.5. Let $G$ be a locally compact group. Then the following statements are equivalent.

(a) $\operatorname{LUC}(G)$ is an injective Banach left $L^{1}(G)$-module.

(b) $L U C(G)$ is an injective Banach left $M(G)$-module.

(c) $G$ is discrete.

Proof. (a) $\Rightarrow$ (b). Suppose that $E$ and $F$ are two left $M(G)$-modules, $T: E \longrightarrow F$ is an admissible left $M(G)$-module monomorphism and $S: E \longrightarrow \operatorname{LUC}(G)$ is a left $M(G)$-module morphism. Then the injectivity of $\operatorname{LUC}(G)$ as a left Banach $L^{1}(G)$ module implies that there exists a left $L^{1}(G)$-module morphism $R: F \longrightarrow \operatorname{LUC}(G)$ with

$$
R \circ T=S
$$


Since $\operatorname{LUC}(G)$ has a left bounded approximate identity $\left(e_{\gamma}\right)_{\gamma \in \Gamma}$ in $L^{1}(G)$, we have

$$
\begin{aligned}
R(\mu \cdot \xi) & =\lim _{\gamma} e_{\gamma} \cdot R(\mu \cdot \xi) \\
& =\lim _{\gamma} R\left(e_{\gamma} \cdot \mu \cdot \xi\right) \\
& =\lim _{\gamma} e_{\gamma} \cdot \mu \cdot R(\xi) \\
& =\mu \cdot R(\xi)
\end{aligned}
$$

for all $\mu \in M(G)$ and $\xi \in F$. So, $R$ is also a left $M(G)$-module morphism.

(b) $\Rightarrow$ (c). Suppose that $\operatorname{LUC}(G)$ is an injective Banach left $M(G)$-module. Then there exists a left $M(G)$-module morphism

$$
\rho_{G}: B(M(G), \operatorname{LUC}(G)) \longrightarrow \operatorname{LUC}(G)
$$

such that $\rho_{G} \circ \Pi_{G}=I_{\mathrm{LUC}(G)}$, where

$$
\Pi_{G}: \operatorname{LUC}(G) \longrightarrow B(M(G), \operatorname{LUC}(G))
$$

is the canonical embedding defined by

$$
\Pi_{G}(h)(\mu)=\mu \cdot h
$$

for all $h \in \operatorname{LUC}(G)$ and $\mu \in M(G)$. Now, consider

$$
Q: L^{\infty}(G) \longrightarrow B(M(G), \operatorname{LUC}(G))
$$

with

$$
Q(f)(\mu)=\mu_{a} \cdot f
$$

for all $f \in L^{\infty}(G)$ and $\mu \in M(G)$, where $\mu_{a}$ is the absolutely continuous part of $\mu$ with respect to the left Haar measure. In particular,

$$
Q(h)(\phi)=\Pi_{G}(h)(\phi)
$$

for all $h \in \operatorname{LUC}(G)$ and $\phi \in L^{1}(G)$. The result will follow if we show that $\rho_{G} \circ Q$ : $L^{\infty}(G) \longrightarrow \operatorname{LUC}(G)$ is projection on $\operatorname{LUC}(G)$; see [6, Theorem 4]. To show that

$$
k:=\rho_{G}\left(Q(h)-\Pi_{G}(h)\right)=0
$$

for all $h \in \operatorname{LUC}(G)$, choose a left bounded approximate identity $\left(e_{\gamma}\right)_{\gamma \in \Gamma} \subseteq L^{1}(G)$ for $\operatorname{LUC}(G)$. Since $k \in \operatorname{LUC}(G)$ and $\rho_{G}$ is a left $M(G)$-module morphism,

$$
k=\lim _{\gamma} e_{\gamma} \cdot k=\lim _{\gamma} \rho_{G}\left(e_{\gamma} \cdot Q(h)-e_{\gamma} \cdot \Pi(h)\right)=0 ;
$$

indeed, for each $\mu \in M(G)$ we have $\mu * e_{\gamma} \in L^{1}(G)$ for all $\gamma \in \Gamma$, and so

$$
\left(e_{\gamma} \cdot Q(h)-e_{\gamma} \cdot \Pi_{G}(h)\right)(\mu)=\left(Q(h)-\Pi_{G}(h)\right)\left(\mu * e_{\gamma}\right)=0 .
$$

(c) $\Rightarrow$ (a). This follows from the fact that $L^{\infty}(G)$ is always an injective Banach left $L^{1}(G)$-module and that $\operatorname{LUC}(G)=L^{\infty}(G)$ when $G$ is discrete; see [2, Theorem 2.4]. 
Let $A$ be a Banach algebra and let us recall that a Banach left $A$-module $F$ is called flat if $F^{*}$ is an injective Banach right $A$-module. Moreover, a locally compact group $G$ is called amenable if there is a positive functional $m \in L^{\infty}(G)^{*}$ with $\|m\|=1$ and $m \cdot \delta_{x}=m$ for all $x \in G$. The class of amenable groups includes all compact groups and all abelian locally compact groups; however, the discrete free group $\mathbb{F}_{2}$ on two generators is not amenable; see [8] for more details.

THEOREM 2.6. Let $G$ be a locally compact group. Then the following statements are equivalent.

(a) $L U C(G)$ is a flat Banach left $M(G)$-module.

(b) $L U C(G)$ is a flat Banach left $L^{1}(G)$-module.

(c) $G$ is amenable.

Proof. (b) $\Leftrightarrow(\mathrm{c})$. Suppose that $G$ is amenable. Then by the classical result of Johnson [5], $L^{1}(G)$ is an amenable Banach algebra; that is,

$$
H^{1}\left(L^{1}(G), E^{*}\right)=\{0\}
$$

for all Banach $L^{1}(G)$-bimodules $E$. So, $\operatorname{LUC}(G)$ is a flat Banach left $L^{1}(G)$-module; this follows from the fact that if $A$ is an amenable Banach algebra, then each Banach left or right $A$-module is flat; see [3, Theorem VII.2.29].

For the converse, suppose that $\operatorname{LUC}(G)$ is flat as a Banach left $L^{1}(G)$-module; that is, $\operatorname{LUC}(G)^{*}$ is injective as a Banach right $L^{1}(G)$-module. An argument similar to the proof of Theorem 2.1 shows that the Banach right $L^{1}(G)$-module $M(G)$ is a retraction of $\operatorname{LUC}(G)^{*}$. Thus $M(G)$ is also an injective Banach right $L^{1}(G)$-module; this is because each retraction of an injective Banach module is injective; see [3, Proposition III.1.16]. Therefore, $G$ is amenable by [2, Corollary 4.7].

(a) $\Leftrightarrow$ (b). Since the inclusion $\theta: L^{1}(G) \rightarrow M(G)$ is a bounded homomorphism and $M(G)$ is a flat Banach left $L^{1}(G)$-module, (a) implies (b); see [9, Proposition 4.18]. To prove the converse, suppose that $\operatorname{LUC}(G)^{*}$ is an injective Banach right $L^{1}(G)$-module. We must to prove that for each Banach right $M(G)$ modules $E$ and $F$, each admissible monomorphism $T \in{ }_{M(G)} B(E, F)$ and each $S \in$ $M(G) B\left(E, \operatorname{LUC}(G)^{*}\right)$, there exists $R \in{ }_{M(G)} B\left(F, \operatorname{LUC}(G)^{*}\right)$ such that

$$
R \circ T=S \text {. }
$$

By (b), there exists a right $L^{1}(G)$-module morphism $R: F \longrightarrow \operatorname{LUC}(G)^{*}$ with $R$ 。 $T=S$. Since the Banach left $L^{1}(G)$-module $\operatorname{LUC}(G)$ has bounded left approximate identity $\left(e_{\gamma}\right)_{\gamma \in \Gamma}$ in $L^{1}(G)$, it follows that

$$
\begin{aligned}
(R(\xi \cdot \mu)-R(\xi) \cdot \mu)(h) & =\lim _{\gamma}(R(\xi \cdot \mu)-R(\xi) \cdot \mu)\left(e_{\gamma} \cdot h\right) \\
& =\lim _{\gamma}\left(R(\xi \cdot \mu) \cdot e_{\gamma}-R(\xi) \cdot \mu \cdot e_{\gamma}\right)(h) \\
& =\lim _{\gamma}\left(R\left(\xi \cdot \mu \cdot e_{\gamma}\right)-R(\xi) \cdot \mu \cdot e_{\gamma}\right)(h) \\
& =0,
\end{aligned}
$$


for all $h \in \operatorname{LUC}(G), \mu \in M(G)$ and $\xi \in F$. This implies that $R$ is a right $M(G)$ module morphism and the proof is complete.

We end this work with the following conjectures for a locally compact group $G$.

Conjecture 2.7. $\operatorname{LUC}(G)^{*}$ is projective as a Banach left $M(G)$-module if and only if $G$ is compact.

Conjecture 2.8. $\operatorname{LUC}(G)^{*}$ is flat as a Banach left $L^{1}(G)$ or $M(G)$-module if and only if $G$ is amenable.

\title{
Acknowledgements
}

The authors thank the Center of Excellence for Mathematics at the Isfahan University of Technology. The authors would like to thank the referee for invaluable comments.

\section{References}

[1] F. Bahrami, R. Nasr-Isfahani and S. Soltani Renani, 'Homological properties of certain Banach modules over group algebras', Proc. Edinb. Math. Soc. (2) 54 (2011), 321-328.

[2] H. G. Dales and M. E. Polyakov, 'Homological properties of modules over group algebras', Proc. Lond. Math. Soc. (3) 89 (2004), 390-426.

[3] A. Ya. Helemskii, The Homology of Banach and Topological Algebras (Kluwer Academic Publishers Group, Dordrecht, 1989).

[4] E. Hewitt and K. Ross, Abstract Harmonic Analysis II (Springer, New York, 1970).

[5] B. E. Johnson, Cohomology in Banach Algebras, Memoirs of the American Mathematical Society, 127 (American Mathematical Society, Providence, RI, 1972).

[6] A. T. Lau and V. Losert, 'Complementation of certain subspaces of $L_{\infty}(G)$ of a locally compact group', Pacific J. Math. 141 (1990), 295-310.

[7] R. Nasr-Isfahani and S. Soltani Renani, 'Character contractibility of Banach algebras and homological properties of Banach modules', Studia Math. 202 (2011), 205-225.

[8] J. P. Pier, Amenable Locally Compact Groups, Pure and Applied Mathematics (John Wiley and Sons, New York, 1984).

[9] M. C. White, 'Injective module for uniform algebras', Proc. Lond. Math. Soc. (3) 73 (1996), $155-184$.

\section{RASOUL NASR-ISFAHANI, Department of Mathematical Sciences, Isfahan University of Technology, Isfahan 84156-83111, Iran e-mail: isfahani@cc.iut.ac.ir}

\author{
SIMA SOLTANI RENANI, Department of Mathematical Sciences, \\ Isfahan University of Technology, Isfahan 84156-83111, Iran \\ e-mail: simasoltani@cc.iut.ac.ir
}

